\title{
Personalized vagino-cervical microbiome dynamics after oral
} probiotics

Chen Chen ${ }^{1,2,3, *,+}$, Lilan Hao ${ }^{1 *}$, Liu Tian ${ }^{1}$, Liju Song ${ }^{1}$, Xiaowei Zhang ${ }^{1}$, Zhuye Jie $^{1,2,3}$, Xin Tong ${ }^{1}$, Liang Xiao ${ }^{1,4,6}$, Tao Zhang ${ }^{1,2,3}$, Xin $\mathrm{Jin}^{1}$, Xun Xu', Huanming Yang ${ }^{1,7}$, Jian Wang ${ }^{1,7}$, Karsten Kristiansen ${ }^{1,3,6}$, Huijue Jia $^{1,2, \dagger}$

1. BGI-Shenzhen, Shenzhen 518083, China;

2. Shenzhen Key Laboratory of Human Commensal Microorganisms and Health Research, BGI-Shenzhen, Shenzhen, China;

3. Department of Biology, Ole MaalØes Vej 5, University of Copenhagen, Copenhagen, Denmark;

4. Shenzhen Engineering Laboratory of Detection and Intervention of human intestinal microbiome, BGl-Shenzhen, Shenzhen, China;

5. China National Genebank, BGI-Shenzhen, Shenzhen 518120, China;

6. BGI-Qingdao, BGI-Shenzhen, Qingdao, 266555, China;

7. James D. Watson Institute of Genome Sciences, Hangzhou, China

* These authors contributed equally to this work.

† To whom correspondence should be addressed: H.J., C.C.

iiahuijue@genomics.cn or chenchen1@genomics.cn 


\begin{abstract}
The vaginal microbiota is presumably much simpler than the gut microbiome, and oral probiotics appear as a promising means to modulate its homeostasis in the general population. Here, 60 women were followed for over a year before, during and after a probiotic containing Lactobacillus rhamnosus GR-1 and L. reuteri RC-14. Shotgun metagenomic data of 1334 samples from multiple body sites did not support colonization of the probiotics to the vagino-cervical microbiome, yet the microbiome was stable in those dominated by Lactobacilli and some individuals have likely benefited from this medication-free intervention.
\end{abstract}

\title{
INTRODUCTION
}

1 Lactobacilli have long been defined as the keystone species of the healthy 2 vaginal microbiota. Lactic acid, hydrogen peroxide, biosurfactants and 3 bacteriocins produced by these microorganisms help maintain the balance of 4 vaginal microenvironment and wards off pathogens. A non-Lactobacillus5 dominated microbial community has also been reported in women without 6 symptoms of vaginosis, and is characterized by strictly anaerobic bacteria,

7 such as Gardnerella, Atopobium, Prevotella and Peptoniphilus, which leads

8 to significant increase in the risk of adverse conditions, including bacterial 9 vaginosis $(\mathrm{BV})$, preterm birth, urinary infections, human immunodeficiency 10 virus (HIV), human papillomavirus (HPV) and other sexually transmitted infections (STIs) $)^{1,2,3,4,5,6,7}$.

Besides fecal transplant and dietary modulation, probiotics have become a major trend for improving gut microbiome health. E.g. for the gastrointestinal tract, gut-brain axis ${ }^{8,9,10,11,12}$. However, just as the vagino-cervical microbiome has received less attention as the gut microbiome, strategies for modulating the vagino-cervical microbiome is also relatively under studied. 
Lactobacillus rhamnosus GR-1 and L. reuteri RC-14 are well-characterized strains as supplementations in female orally consumed probiotic products. The strains have also been reported to relieve colitis and osteoporosis in animal models ${ }^{13,14}$. However, evidence of their oral administration efficacy in the prevention and treatment of vaginal infection conditions, such as BV, HIV, HPV, Group B Streptococcus (GBS), remains highly debated 15,16,17,18,19. Moreover, the route of oral administrated probiotics to the vagina and their colonization in the multi-site of the human commensal microbiota remains largely unexplored.

Here, we conducted a longitudinal study of 60 women to explored the effect of prolonged probiotics consumption on the vagino-cervical microbiome. To investigate the dynamic alternation of muti-site microbiota after taking the live probiotic capsules, the tongue coat, buccal mucosal and fecal microbiome composition were also analyzed.

\section{RESULTS}

\section{Demographic characteristics of the cohort}

In our cohort, 60 healthy women were recruited (median age 31, 95\% confidence interval (CI) 30-34; Supplementary Table 1). Samples were initially collected 300 days before the intervention phase. The relations of time points (before: B2, B1, B0; during: O1-O5; after: W1, W2), quantity of capsules and menstrual cycle were showed (Figure 1, Supplementary Table 2). Vagino-cervical samples were collected at all the time points. Other multisite samples including buccal mucosa samples, tongue coat samples and fecal samples were collected at eight of the time points (B0-W2). All the samples were self-collected referring to a self-collection protocol, and performed the metagenomic analysis with shotgun sequencing data (Supplementary Figure 1). The microbial reads were extracted by filtering the 
human reads and subsequently used for taxonomic profiling of the microbiome (Supplementary Table 3).

\section{Lack of oral probiotic colonization in the vagino-cervical microbiome}

Our data showed both two probiotics were hardly present in all the body sites even during intervention period (Supplementary Figure 2a, 2b). The exception was L. rhamnosus GR-1 in fecal samples, which showed a weak colonization in the time-point $\mathrm{O} 4$ compared to the baseline $(P=0.01$ but $q>0.05$, Supplementary Figure 2b). Likewise, almost no change in the Shannon diversity index and Bray-Curtis dissimilarity were found between baseline and the probiotics period (Supplementary Figure 2c, 2d). We also collected the vaginal $\mathrm{pH}$ accompanying the sampling, no significant differences of vaginal $\mathrm{pH}$ were detected in all the time points $(P=0.87$, Supplementary Figure $2 \mathrm{e})$. Together, this probiotics supplementation may be limited colonization in vaginal or oral sites.

\section{A stable vagino-cervical microbiome is resilient against Lactobacilli intake}

A previous study of the oral probiotics in individuals with BV was preceded by the antibiotic metronidazole treatment ${ }^{20}$, it is not clear in a more general, subclinical setting, whether the probiotic strains could really be recommended for anyone with a slight discomfort or who tested positive for potential pathogens. Compared to metagenomic data from the previous year, we classified the subjects into two groups: dysbiosis and stable, using the Bray-Curtis dissimilarity index (defined as the median BrayCurtis dissimilarity between B2/B1 to B0) (Figure 2a). As expected, individuals of the stable group were dominated by Lactobacillus genera and displayed persistently lower Bray-Curtis distances, $\mathrm{pH}$, Shannon alpha diversity over time compared to that of individuals in dysbiosis group (Figure $2 \mathrm{~b}-2 \mathrm{e})$. Thus, exogenous probiotics bacteria may be limited in 
impacting the vagino-cervical microbiome in stable group. However, there was still limited efficacy of probiotics in dysbiosis group (Figure2c, 2d). Of note, fecal microbiome of women in the dysbiosis group were also detected a less diverse but changed markedly compare to stable group (Supplementary Figure 3).

To evaluate the health condition of an independent sample, we then constructed a cross-validated random-forest model based on the vaginocervical microbiome of the two groups (Figure 2f). 6 bacterial species included in the classifier, Gardnerella vaginalis, Ureaplasma unclassified and Prevotella bivia were significantly enriched in the dysbiosis group (Figure $2 \mathrm{~g}$ ). We therefore classified samples using this model. In total, 244 dysbiosis samples (Dy_s) and 166 stable samples (St_s) were classified in this cohort. To be expected, St_s were almost dominated by L. crispatus, $L$. iners and L. jensenii (Figure 2h, Supplementary Figure 4). The type transitions of samples within subjects displayed a high level of stability longitudinally, and showed no drastically transition from Dy_s to St_s during and after probiotics supplementation compared to their baselines (Figure 3, Supplementary Figure 5). Taken together, these findings point out that women consumption of the probiotics results no shedding in vagina and had no apparent effects on re-establishing a beneficial vaginocervical microbiome.

\section{Dynamics of Personalized vagino-cervical Microbiome}

The vagino-cervical microbiome of 60 women were visualized by mapping temporal dynamics in community composition longitudinally (Figure 4). The microbiome composition of subjects in stable group appeared to be comparatively stable over time, and were typically dominated by $L$. iners, $L$. crispatus or $L$. jensenii. In these women, the slightly transitions were mostly exhibited among the different Lactobacillus species. The relative 
abundance of non-Lactobacillus only resides in a small space, and showed little need to improve the vagino-cervical microbial ecosystem by consumption of the probiotics. The microbiome composition of subjects in dysbiosis group changed markedly and continuously over time. However, the relative abundance of Lactobacillus was observed increased during and after probiotics supplementation only in 4 subjects, including $L$. crispatus in S020, L. iners in S030, Lactobacillus acidophilus in S025, and L. iners and Lactobacillus sp. 7_1_47FAA in S065 (Figure 5a-5d, Supplementary Figure 6). All the aforementioned Lactobacillus were present as the endogenous bacteria from the baseline period except $L$. acidophilus (Supplementary Figure 6). These results suggested that endogenous vaginal Lactobacilli could increase after the oral probiotics

Subjects S020, S030and S013 were detected to be infected with HPV in the baseline, but gradually be cleared away during and after their probiotic supplementation (Figure 5a, 5b, 5e). Interestingly, with the clearance away of the HPV, Bifidobacterium including B. bifidum and $B$. dentium were harboured as the dominated genus in subject S013 (Figure 5e). HPV infections were also detected in fecal samples of this subject, with a similar trend of vaginal samples in the same individual (Figure $5 \mathrm{e}$ ). These results suggested that supplementation of these two probiotics may had some effects on HPV clearance. Streptococcus agalactiae (Group B Streptococcus), a bacterium responsible for neonatal sepsis and recently reported in placenta ${ }^{21}$, could be detected in $16.7 \%$ of the subjects. But the rate of vaginal $S$. agalactiae colonization did not differ significantly between baseline and the probiotics period $(P=0.98$, Supplementary Figure 7), consistent with colonization effects in pregnancy ${ }^{19}$.

\section{DISCUSSION}

61 In this study, we provided metagenomic data for the first time following oral 
62 probiotics supplementation. Although some volunteers showed the 63 Lactobacilli probiotic strains in the fecal samples, there was no increase in the 64 probiotic strains in the vaginal or oral sites, suggesting that L. rhamnosus GR651 and $L$. reuteri GR-14 were not translocated from the gut to the vagina ${ }^{22}$. 66 PCR evidence of vaginal colonization has been reported for these strains for 67 individuals with $\mathrm{BV}$, and our metagenomic data raise the possibility that 68 endogenous vaginal Lactobacilli (L. crispatus, L. iners, etc.) have been 69 promoted by the oral probiotics through immunological or metabolic 70 modulation ${ }^{23}$. We present the efficacy results from a comprehensive view of 71 dysbiosis in the vagino-cervical microbiome. In volunteers with a Lactobacilli72 dominated vagino-cervical microbiome, the microbiome is largely unchanged over one year, whether during probiotic intake or not. The dysbiosis group 74 have a more diverse vagino-cervical microbiome and a less diverse fecal 75 microbiome, but $\mathrm{pH}$ and microbiome dynamics varied between individuals. 76 Without better ways of minimizing the individual dynamics, a much larger 77 cohort would be needed to further analyze and predict the effects of probiotics 78 supplementation.

It remains possible that $L$. crispatus could be more effective as an oral probiotic for the vagino-cervical microbiome. Other factors such as hormonal dynamics ${ }^{24}$, seasonal changes ${ }^{25}$ may also have influenced our study. Recent studies of vaginal microbial transplant (VMT) and treatment of BV using L. crispatus have all used a more direct topical application after standard metronidazole treatment ${ }^{26,27}$. Yet, oral probiotics are more readily consumed in a subclinical setting, and may be more acceptible for pregnant women with a risk for preterm birth.

\section{Online content}


80

81

82

83

84

Any methods, additional references, Nature Research reporting summaries, source data, statements of data availability and associated accession codes are available at https://db.cngb.org/search/project/CNP0001123.

\section{REFERENCES}

1. Onderdonk, A. B., Delaney, M. L. \& Fichorova, N. The Human Microbiome during Bacterial Vaginosis. 29, 223-238 (2016).

2. Fettweis, J. M. et al. The vaginal microbiome and preterm birth. Nat. Med. 25, 1012-1021 (2019).

3. DiGiulio, D. B. et al. Temporal and spatial variation of the human microbiota during pregnancy. Proc. Natl. Acad. Sci. 112, 11060-11065 (2015).

4. Klatt, N. R. et al. Vaginal bacteria modify HIV tenofovir microbicide efficacy in African women. Science (80-. ). 356, 938-945 (2017).

5. Usyk, M. et al. PLOS PATHOGENS Cervicovaginal microbiome and natural history of HPV in a longitudinal study. 1, 1-20 (2020).

6. Mitra, A. et al. The vaginal microbiota, human papillomavirus infection and cervical intraepithelial neoplasia $\square$ : what do we know and where are we going next $\square$ ? Microbiome 1-15 (2016) doi:10.1186/s40168-016-0203-0.

7. Anahtar, M. N., Gootenberg, D. B., Mitchell, C. M. \& Kwon, D. S. Review Cervicovaginal Microbiota and Reproductive Health $\square$ : The Virtue of Simplicity. Cell Host Microbe 23, 159-168 (2018).

8. Markey, K. A., Brink, M. R. M. Van Den \& Peled, J. U. Forum Therapeutics Targeting the Gut Microbiome $\square$ : Rigorous Pipelines for Drug Development. Cell Host Microbe 27, 169-172 (2020).

9. Zeevi, D. et al. Personalized Nutrition by Prediction of Glycemic Article Personalized Nutrition by Prediction of Glycemic Responses. 1079-1094 (2015) doi:10.1016/j.cell.2015.11.001.

10. Suez, J., Halpern, Z., Segal, E. \& Elinav, E. Personalized Gut Mucosal Colonization Resistance to Empiric Probiotics Is Associated with Unique Host 
and Microbiome Features Article Personalized Gut Mucosal Colonization

Resistance to Empiric Probiotics Is Associated with Unique Host and Microbiome Feat. Cell 174, 1388-1405.e21 (2018).

11. Henriques, F., Ribeiro, C. \& Ezra-nevo, G. ScienceDirect The diet-microbiome tango $\square$ : how nutrients lead the gut brain axis. 122-132 (2020) doi:10.1016/j.conb.2020.02.005.

12. Johnson, A. J. et al. Daily Sampling Reveals Personalized Diet- Microbiome Associations in Humans Article Daily Sampling Reveals Personalized DietMicrobiome Associations in Humans. 789-802 (2019) doi:10.1016/j.chom.2019.05.005.

13. Britton, R. A. et al. Probiotic L. reuteri treatment prevents bone loss in a menopausal ovariectomized mouse model. J. Cell. Physiol. 229, 1822-1830 (2014).

14. Mccabe, L. R., Irwin, R., Schaefer, L. \& Britton, R. A. Probiotic use decreases

15. Hummelen, R. et al. Lactobacillus rhamnosus GR-1 and L. reuteri RC-14 to

17. Ou, Y.-C. et al. The influence of probiotics on genital high-risk human

18. Yang, S. et al. Effect of Oral Probiotic Lactobacillus rhamnosus GR-1 and

19. Sharpe, M. et al. Effectiveness of oral intake of Lactobacillus rhamnosus GR-1 and Lactobacillus reuteri RC-14 on Group B Streptococcus colonization during 
pregnancy: a midwifery-led double-blind randomized controlled pilot trial. $J$. Matern. Fetal. Neonatal Med. 1-8 (2019) doi:10.1080/14767058.2019.1650907.

20. Anukam, K. et al. Augmentation of antimicrobial metronidazole therapy of bacterial vaginosis with oral probiotic Lactobacillus rhamnosus GR-1 and placebo controlled trial. 8, 1450-1454 (2006).

21. de Goffau, M. C. et al. Human placenta has no microbiome but can contain potential pathogens. Nature (2019) doi:10.1038/s41586-019-1451-5.

22. Marrazzo, J. M. et al. Extravaginal reservoirs of vaginal bacteria as risk factors for incident bacterial vaginosis. J. Infect. Dis. 205, 1580-8 (2012).

23. Cervantes-Barragan, L. et al. Lactobacillus reuteri induces gut intraepithelial CD4+CD8 $\alpha \alpha+$ T cells. Science (80-. ). 357, 806-810 (2017).

24. Gajer, P. et al. Temporal Dynamics of the Human Vaginal Microbiota. Sci. Transl. Med. 4, 132ra52-132ra52 (2012).

25. Smits, S. A. et al. Seasonal cycling in the gut microbiome of the Hadza huntergatherers of Tanzania. Science (80-. ). 357, 802-805 (2017).

26. Lev-sagie, A. et al. Vaginal microbiome transplantation in women with

27. Morris, S. et al. Randomized Trial of Lactin-V to Prevent Recurrence of

28. Chen, C. et al. The microbiota continuum along the female reproductive tract and its relation to uterine-related diseases. Nat. Commun. 8, 875 (2017).

29. Fang, C. et al. Assessment of the cPAS-based BGISEQ-500 platform for metagenomic sequencing. 1-8 (2018) doi:10.1093/gigascience/gix133.

30. Han, M. M. et al. A novel affordable reagent for room temperature storage and transport of fecal samples for metagenomic analyses. Microbiome 6, 43 (2018).

31. Pan, H. et al. A gene catalogue of the Sprague-Dawley rat gut metagenome. Gigascience 7, (2018). 
169 33. Schmieder, R. \& Edwards, R. Fast identification and removal of sequence

$170 \quad$ contamination from genomic and metagenomic datasets. PLoS One (2011)

171 doi:10.1371/journal.pone.0017288.

172 34. Zaharia, M. et al. Faster and More Accurate Sequence Alignment with SNAP.

$173 \quad$ (2011).

174 35. Truong, D. T. et al. MetaPhlAn2 for enhanced metagenomic taxonomic

175 profiling. Nat. Methods 12, 902-903 (2015).

176 36. Hao, Y. et al. HPViewer: Sensitive and specific genotyping of human

177 papillomavirus in metagenomic DNA. Bioinformatics 34, 1986-1995 (2018).

\section{Acknowledgements}

180 This research was supported by the Shenzhen Municipal Government of 181 China (JCYJ20170817145523036). The authors are very grateful to 182 colleagues at BGI-Shenzhen for sample collection, and discussions, and 183 China National Genebank (CNGB) Shenzhen for DNA extraction, library 184 construction, sequencing.

\section{Author contributions}

H.J. and C.C. conceived and organized this study. C.C., L.H., L.S., and X.Z. performed the sample collection and questionnaire collection. L.H., C.C., L.T, and Z.J. performed the bioinformatic analyses, H.J., C.C. and X.Z. wrote the manuscript. All authors contributed to data and texts in this manuscript.

\section{Competing interests}

The authors declare no competing financial interest. 


\section{FIGURE LEGENDS}

\section{Fig. 1. Sampling strategy of the cohort.}

197 We followed 60 healthy women for over one years each. The samples were 198 classified into 10 time points: baseline (B2, B1, B0), during intervention period 199 (O1-O5) and at the end of the intervention (W1, W2), according to the 200 sampling time, quantity of capsules and menstrual cycle.

Fig. 2. The vagino-cervical microbiome characteristics in dysbiosis and stable groups.

204 a, b, c, d, e. The 46 subjects who had complete baseline time points (B2, B1, B0) were classified into two groups: dysbiosis (red) and stable (blue). a. 206 Group the subjects according to the Bray-Curtis dissimilarity. Purple dots, 207 distance between B2 and B0; green dots, distance between B1 to B0. stable 208 group, both dots in subjects were lower than their corresponding median 209 Bray-Curtis distance (purple line: B2-B0; green line: B1-B0). Others were 210 classified into dysbiosis group. b. The Bray-Curtis distance at each time point 211 relative to $\mathrm{B} 0$. Boxplots show median and lower/upper quartiles; whiskers 212 show inner fences. Wilcoxon ranked sum test was used to conduct 213 comparisons between two groups in each time point, an asterisk denotes q $214<0.05$, two asterisks denote $q<0.01$, three asterisks denote $q<0.001$, four 215 asterisks denote $\mathrm{q}<0.0001$. The Relative abundance of Lactobacillus spp. (c), 216 vaginal $\mathrm{pH}(\mathbf{d})$, and Shannon diversity index (e) were compared between two 217 groups. Kruskal-Wallis test was used to conduct temporal dynamics 218 comparisons within groups. f. Microbiome-based discrimination between 219 dysbiosis and stable groups. Receiver operating characteristic curve (ROC) according to 138 baseline samples (B2, B1, B0) from 27 dysbiosis subjects

221 and 19 stable subjects calculated by cross-validated random forest models. 222 Area under ROC (AUC) and the 95\% confidence intervals are also shown. $\mathbf{g}$. 
224 the models. The color of each species indicates its enrichment in Dy_s (red)

225 or St_s (blue) or no significant direction (black), respectively. $\mathbf{h}$. PCoA on the

226 Dy_s and St_s based on Bray-Curtis distance. Enterotype information was

227 shown in Supplementary Figure 2.

228

Fig. 3. Temporal dynamics of vagino-cervical microbiome before, during and after oral probiotics.

231 Color bar indicating dysbiosis subjects, stable subjects and unclassified 232 subjects. Subject IDs are indicated on the left. b. Profiles of Dy_s or St_s 233 samples for 60 subjects before, during and after oral probiotics. Each shape 234 (hollow square, solid square or diamond) represents one sample in the time 235 series. c. Box plot of Bray-Curtis dissimilarity between all pairs of samples 236 within each subject. d. Box plot of Shannon diversity index of samples within 237 each subject. Boxplots show median and lower/upper quartiles; whiskers 238 show inner fences (c and $\mathbf{d}$ ).

Fig. 4. Dynamics of personalized vagino-cervical microbiome. Heatmaps of the main taxa at species levels in 60 subjects is shown. Dysbiosis group, stable group and unclassified subjects present in three lines.

Fig. 5. Vagino-cervical microbiome in five selected subjects.

The microbial composition in each vagino-cervical sample at the species level according to MetaPhIAn2 is shown in the top. Vaginal and stool HPV types below the bar graphs were identified by HPViewer. RPKM is the abbreviation of "Reads Per Kilobase per Million reads". Samples types including Dy_s (red) and St_s (blue). Other characteristics of subjects including quantity of capsules, capsule time, menstruation, vaginal $\mathrm{pH}$, sensation changes, medical information, sexual intercourse and vaginal douching is shown in the bottom of the table. 


\section{Methods}

\section{Cohort demographics}

255 With the baseline for the vagino-cervical microbiome studied from May 2017 256 and Feb. 2018, we started the metagenomic study for oral probiotics 257 supplementation over the course of 3 months, followed by a two-month wash 258 out period. The commercial probiotic capsules containing Lactobacillus 259 rhamnosus GR-1 and Lactobacillus reuteri GR-14, and each capsule at 2.5 260 billion colony forming units (CFUs). The study was approved by the 261 Institutional Review Boards at BGI-Shenzhen (IRB approval numbers 17244). 26260 healthy women aged from 23 to 61 were recruited in Shenzhen, China 263 (Supplementary Table 1). Exclusion criteria included: (i) Pregnant women, (ii) consumption of probiotics or antibiotics in any form within one month prior to participation. All participants provided written informed consent at enrolment, and then received a first online questionnaire covering comprehensive demographic characteristics (Supplementary Table 1). The study design consisted of three phases, baseline (10 months), probiotic intervention (consumed 90 capsules of probiotics) and follow-up (2 months). Samples were collected three times during the baseline phase (B2-B0). Time point B2 was about 10 months before probiotic intervention, and B1 was about 1.5 months before probiotic intervention. B0 was the most recent time point, 273 participants were instructed to collect samples after menses period, then began to received probiotic capsules. During the intervention phase, each participant was assigned 90 capsules of probiotics and instructed to take one capsule daily. Samples were scheduled 7 (O1), 14 (O2) days after intervention, then monthly after menses period throughout the rest of the 278 intervention (O3, O4, O5). After intervention, two follow-up visits were 279 scheduled monthly after menses period (W1, W2). Vaginal samples were collected at each time point using a home collection kit. Two vaginal swabs were requested, the swab head of one was put into tube with storage reagent 
282 (ref), the other one was brushed on the $\mathrm{pH}$ test strips. Other three different 283 kinds of samples (buccal mucosa samples, tongue coat samples and fecal 284 samples) were also collected by self-sampling at all time points except B2, B1. 285 Participants were also requested to fill in an online questionnaire at each time 286 point. The information of questionnaire including vaginal $\mathrm{PH}$ value, sampling 287 time, menstruation, sexual activity. Samples belonged to probiotic intervention 288 were removed when the participant's average capsule of probiotics was less 289 than 0.5 a day. Throughout the entire study 1334 samples including 322 290 tongue coat samples, 263 buccal mucosa samples, 436 vaginal samples and 291313 fecal samples were collected.

\section{DNA extraction and metagenomic shotgun sequencing}

293 DNA extraction of all samples from four body sites was performed as 294 described $^{28}$. Metagenomic shotgun sequencing was performed on the 295 BGISEQ-500 platform (100bp of paired-end reads) ${ }^{29-32}$. The sequencing 296 reads of stool samples were quality-controlled using Overall Accuracy (OA) 297 control strategy (https://github.com/Scelta/OAFilter), and then aligned to hg19 298 to remove human reads using SOAP2.22 (SOAPaligner/soap2, 299 RRID:SCR_005503) as described previously ${ }^{29}$. Stringent condition for 300 removal of host sequences was used for tongue coat samples, buccal 301 mucosa samples and vaginal samples ${ }^{32}$, through alignment to the hg19, hg38 302 and $\mathrm{YH}$ reference by DeconSeq ${ }^{33}$ (version 0.4 .33 ) and SNAP ${ }^{34}$. Taxonomic 303 assignment of the high-quality metagenomic shotgun data of samples from 304 four body sites were performed using MetaPhIAn2 ${ }^{35}$ version 2.7.0 with 305 database v20. HPViewer with the default parameters was used to detect 306 genotyping of HPV in the high-quality metagenomic sequencing data of 307 samples $^{36}$. 


\section{Statistical analysis}

310 Alpha diversity and beta diversity were calculated on species relative 311 abundances using Shannon-Wiener index and Bray-Curtis dissimilarity, 312 respectively. Kruskal-Wallis test was used to make temporal dynamics 313 comparisons among different time points, including species relative 314 abundance of oral probiotics, Shannon-Wiener index, Bray-Curtis dissimilarity, 315 vaginal $\mathrm{pH}$ and relative abundance of Lactobacillus genera. Wilcoxon rank 316 sum (Mann-Whitney $U$ ) and Wilcoxon signed-rank test were used to make 317 comparisons between two groups or each of two time points. The statistical 318 significance was with a p value threshold of 0.05 and a false discovery rate 319 (FDR) threshold of $\mathrm{q}<0.05$.

320 To build a predictive model to identify microbial dysbiosis, the species relative 321 abundances in the baseline samples were calculated with the training set with 322500 trees in the random Forest package (version 4.6-14). Five-fold cross323 validation was performed five times. The cross-validation error curves from 324 the five trials were averaged, and the minimum error in the averaged curve 325 plus the standard deviation at that point were used as the cutoff for acceptable error. From the sets of species with a classification error less than the cutoff, the set with the smallest number of species was chosen as the optimal set, as in previous methods on the vagino-uterine microbiome ${ }^{28}$. Relative abundances of species in all 410 vaginal samples were used to determine the optimal community types of the vagino-uterine microbiome according to hierarchical clustering based on the Jensen-Shannon distances and Ward linkage. And more statistical details were described in the results and denoted in figure legends, including sample summary, distribution, the statistical method and the statistical test used and significance. 
bioRxiv preprint doi: https://doi.org/10.1101/2020.06.16.155929; this version posted June 17, 2020. The copyright holder for this preprint (which was not certified by peer review) is the author/funder. All rights reserved. No reuse allowed without permission.

336 Data availability

337 Metagenomic shotgun sequencing data for all samples have been deposited

338 to the (CNGB) database under the accession code CNP0001123.

339 


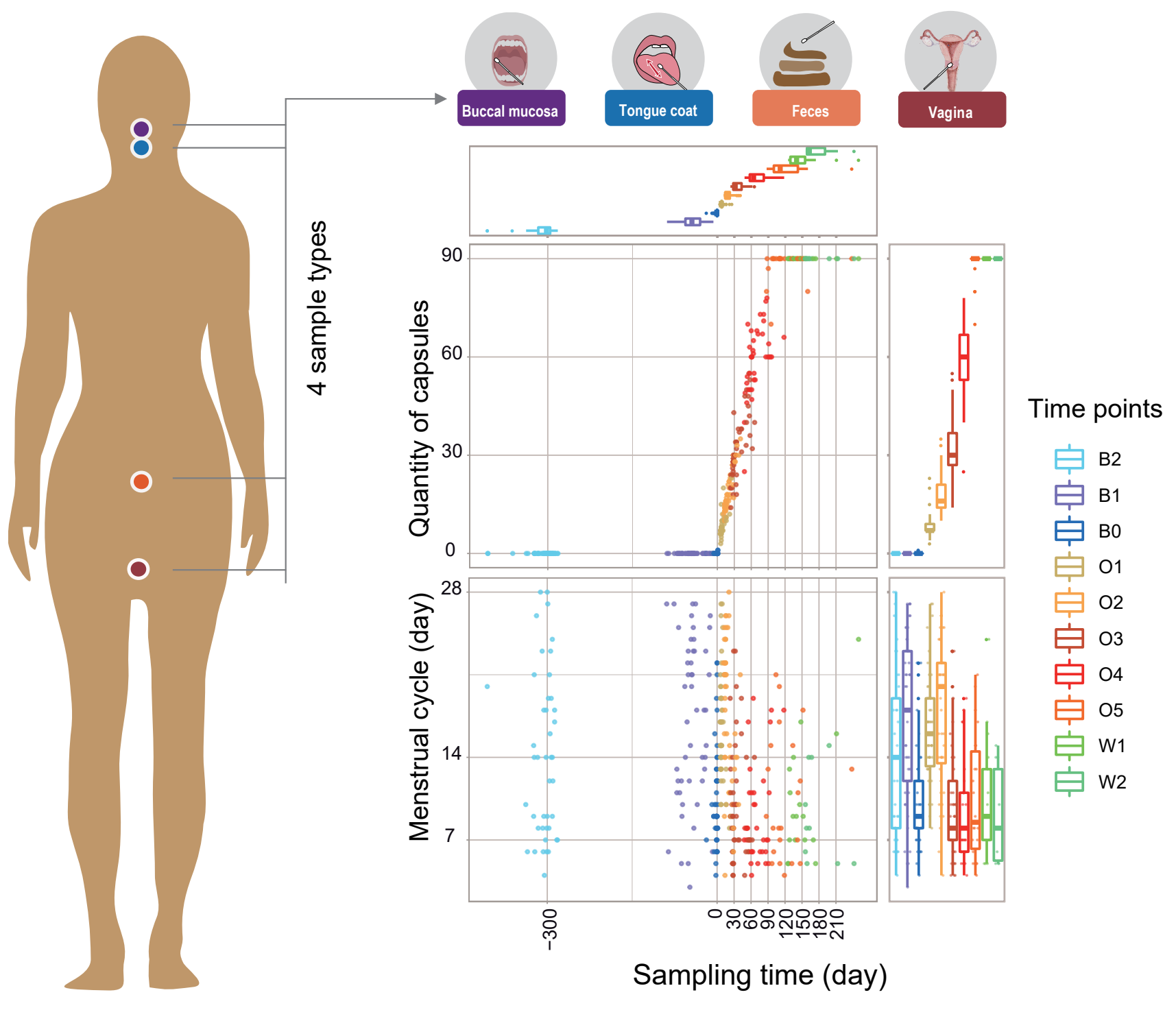


$1.00 \mid$

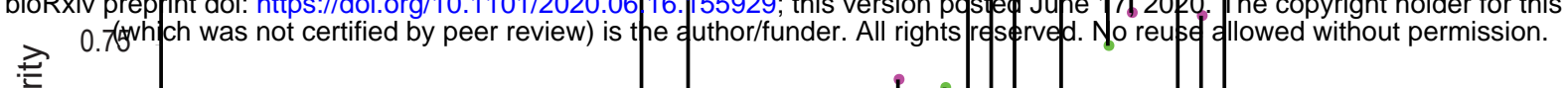

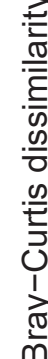

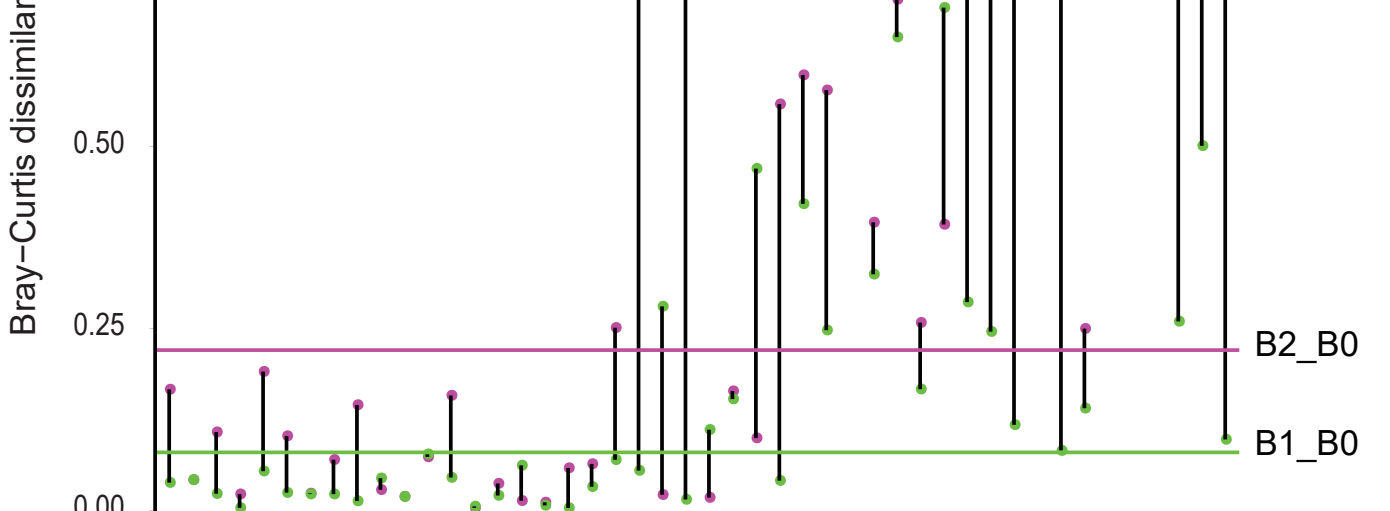

\begin{tabular}{l|l}
$\mathrm{b}$ & Kruskal-Wallis, $p=0.083$
\end{tabular}

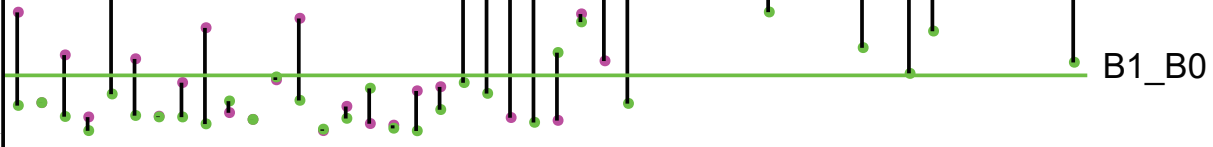
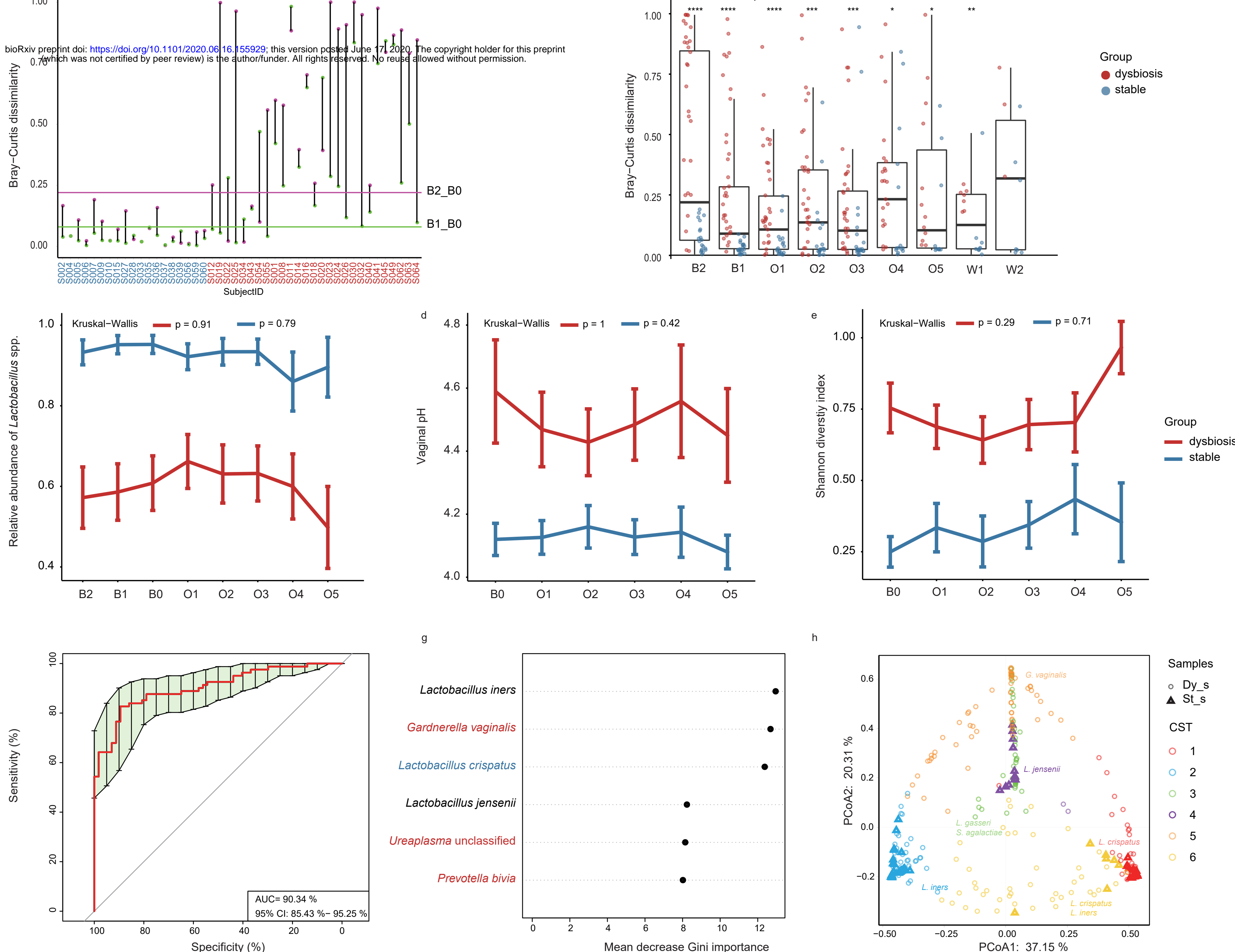

Samples

- Dy_s

CST

○ 1

$\circ 2$

3 



1
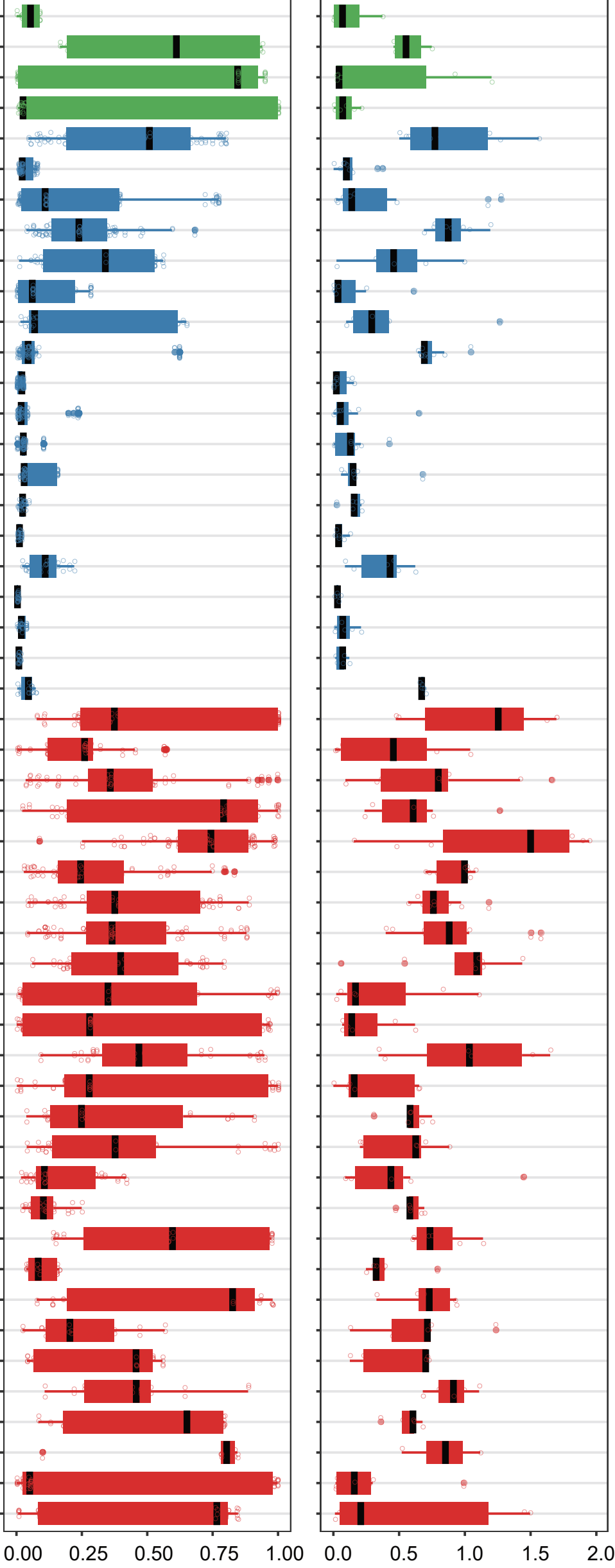

Bray-Curtis dissimilarity

Shannon diversity index 

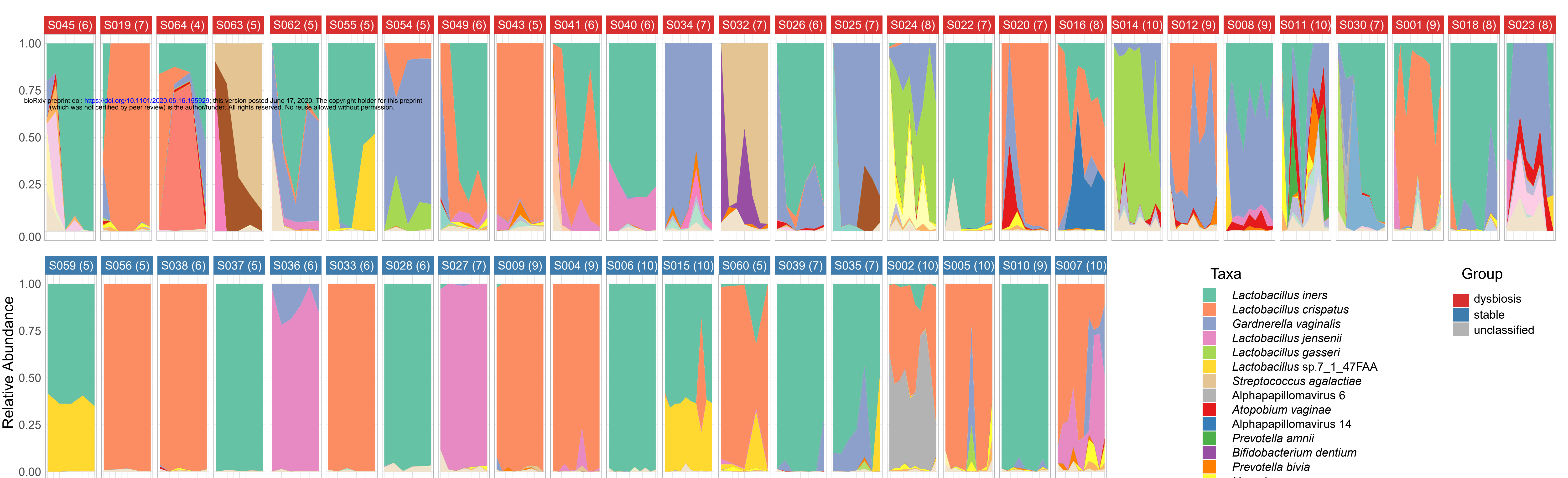

Taxa

Group

Lactobactus iners

Lactobacillus crispatus

Gactoballus jenseni

Lactobacillus gasse

actobacillus sp.7_1_47FAA

treptococcus agalactia

Alphapapillomavirus

Atopobium vaginae

Alphapapillomavirus

Prevotella bivia

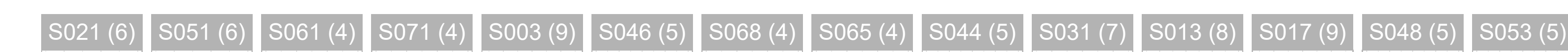

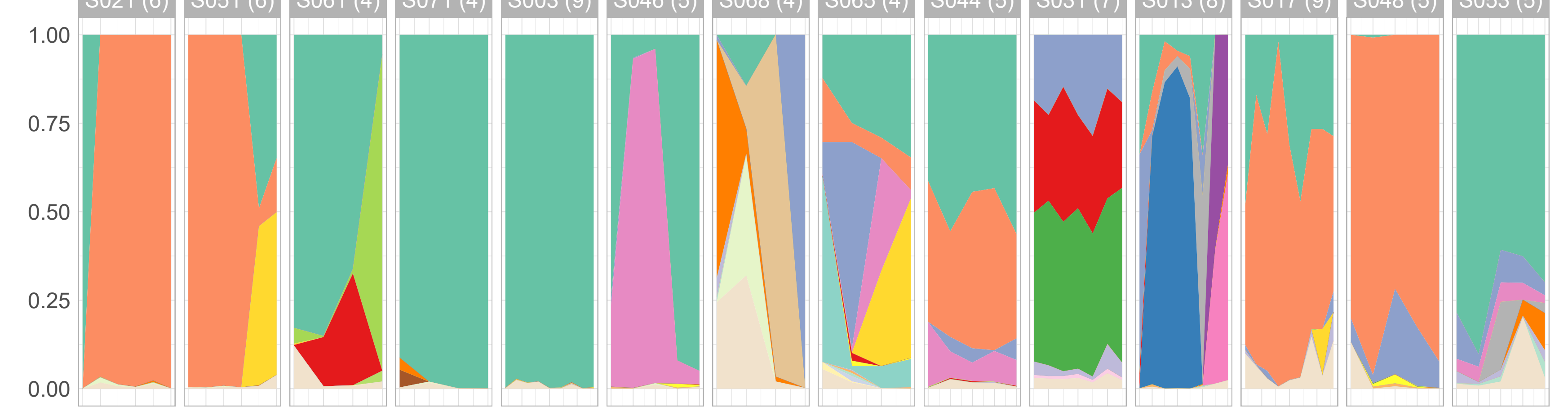

Lactobacillus acidophilus

Bifidobacterium bifidum

Chlamydia trachomatis

Provotella timonensis

Alphapapillomavirus 10

Ureaplasma unclassified

Escherichia coli

Megasphaera genomosp type

Prevotella disiens

Lactobacillus johnsonii

Ureaplasma urealyticum

Caulobacter unclassified

other 

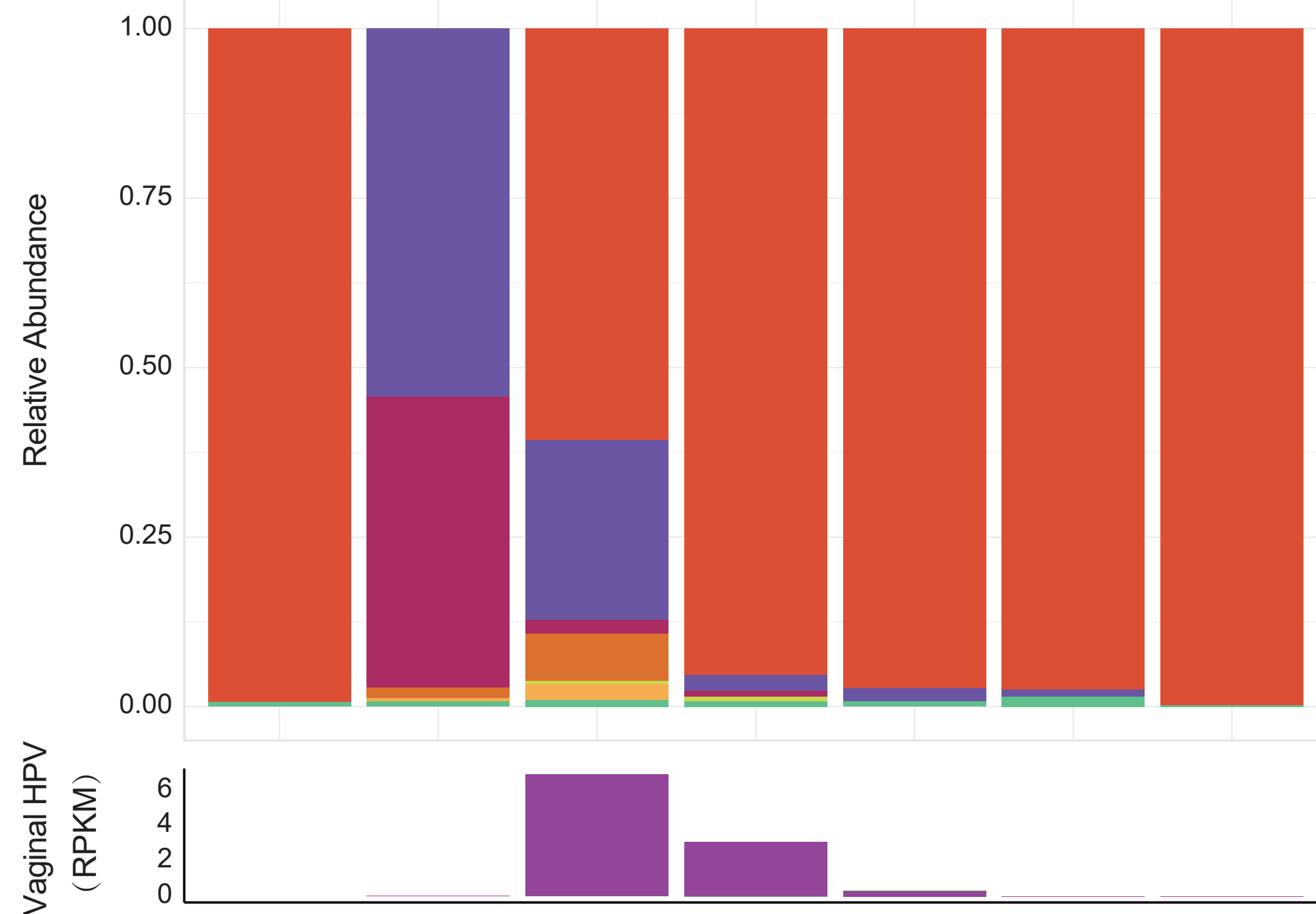$$
\begin{aligned}
& 6 \\
& 4 \\
& 0 \\
& 0
\end{aligned}
$$

Sample types

Quantity of capsule capsule_time (month)

\begin{tabular}{l} 
Menstruation \\
Vaginal $\mathrm{pH}$ \\
\hline
\end{tabular}

Sensation change

Taking medications within 1 month

Vaginal douching within1monts

C S025

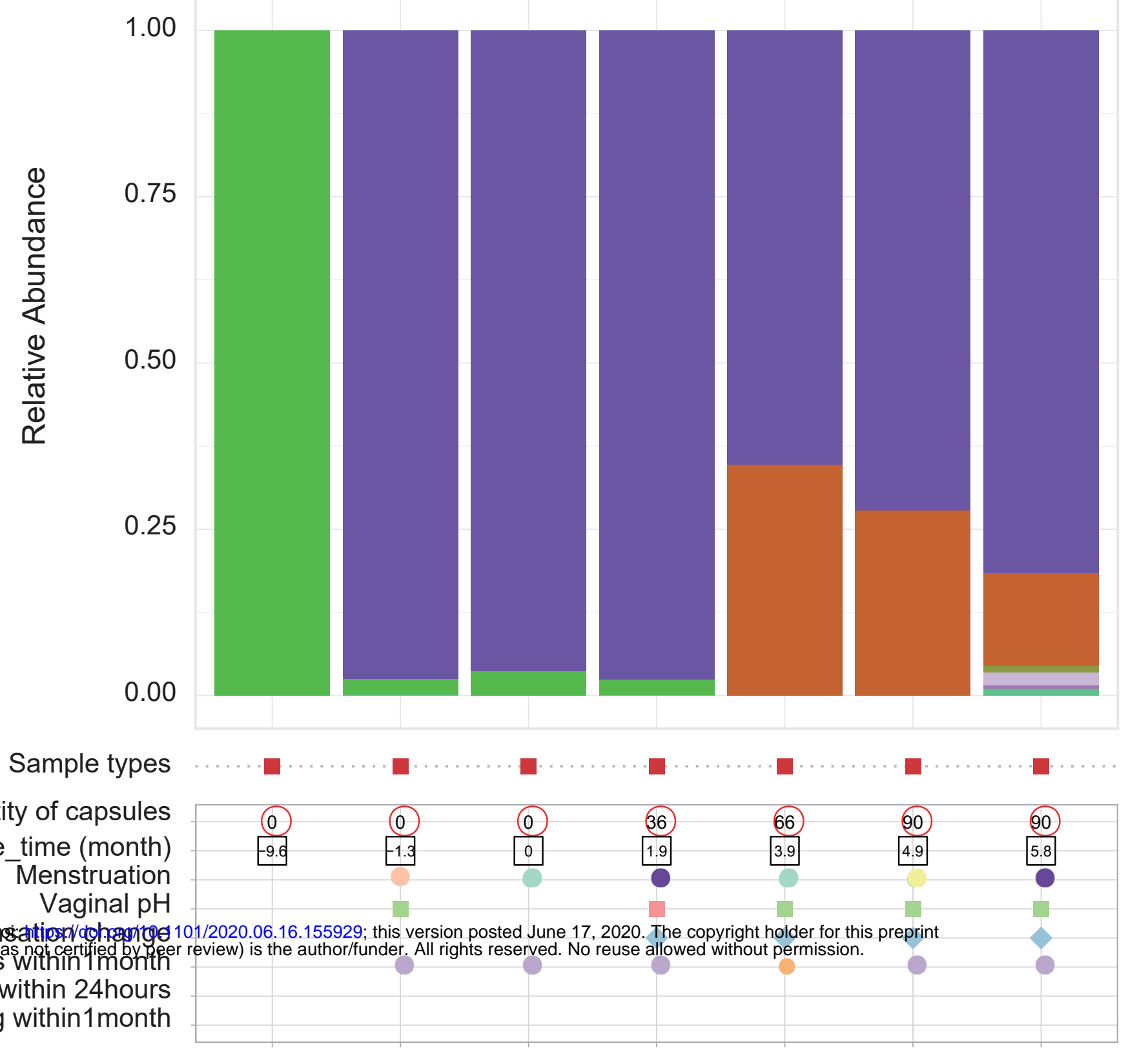

S030
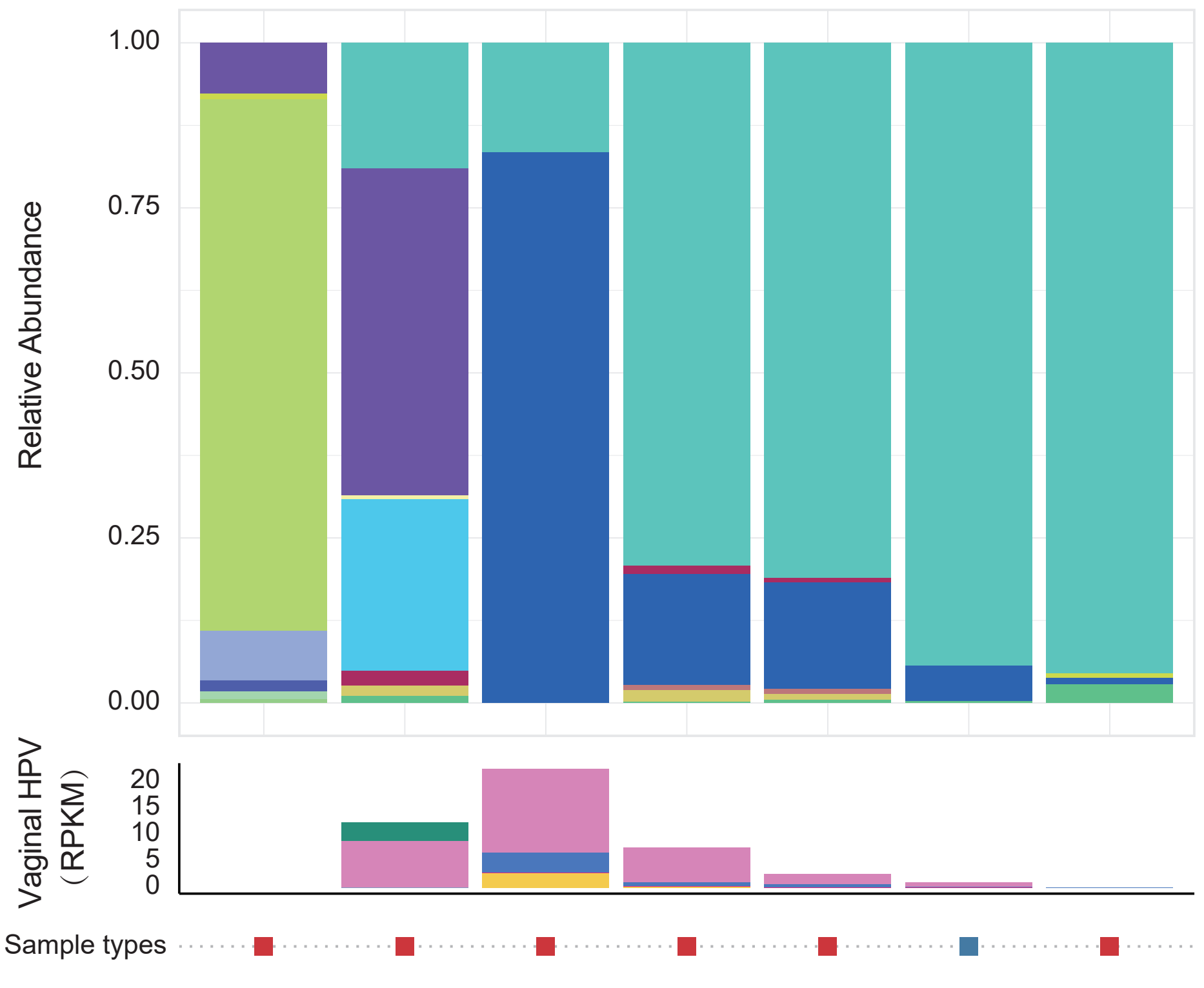

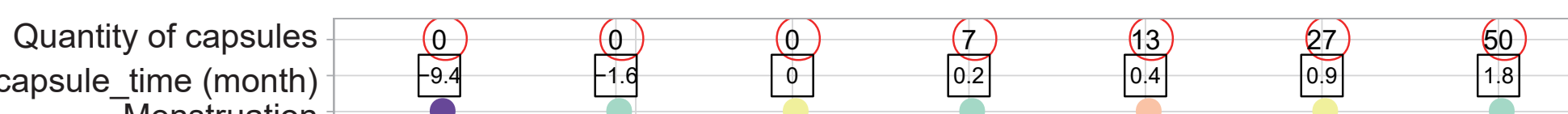

Menstruation
Vaginal pH
pH

Taking medications within 1 month Sexual intercourse within 24 hours Vaginal douching within 1 hours

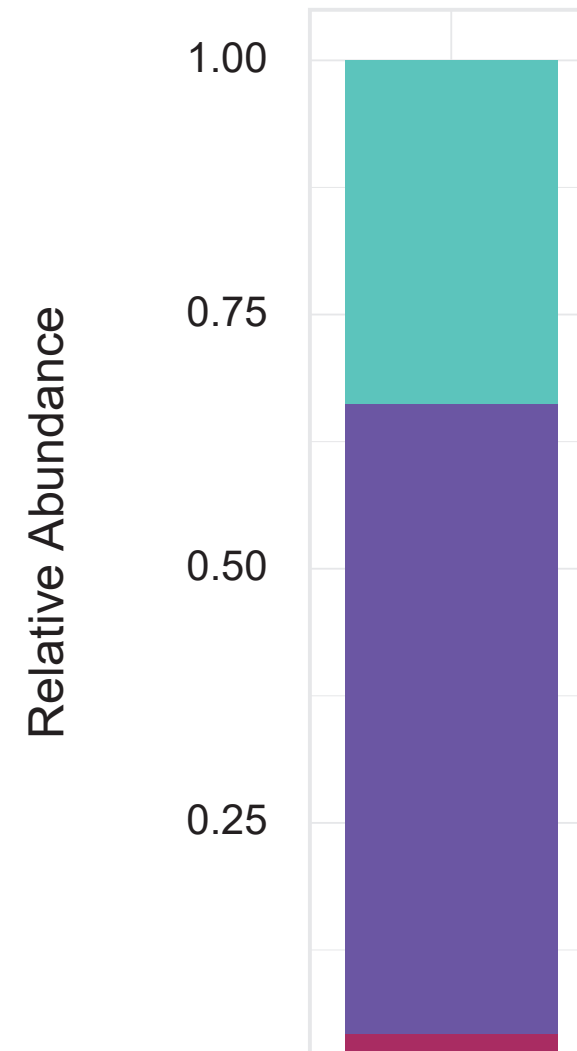

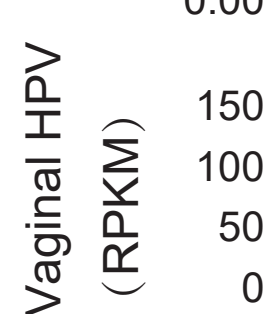

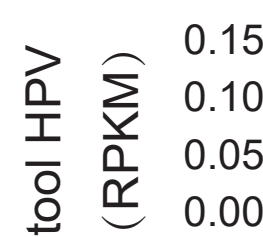

Sample types

Quantity of capsules capsule_time (month)

Vaginal $\mathrm{pH}$

Sensation change d

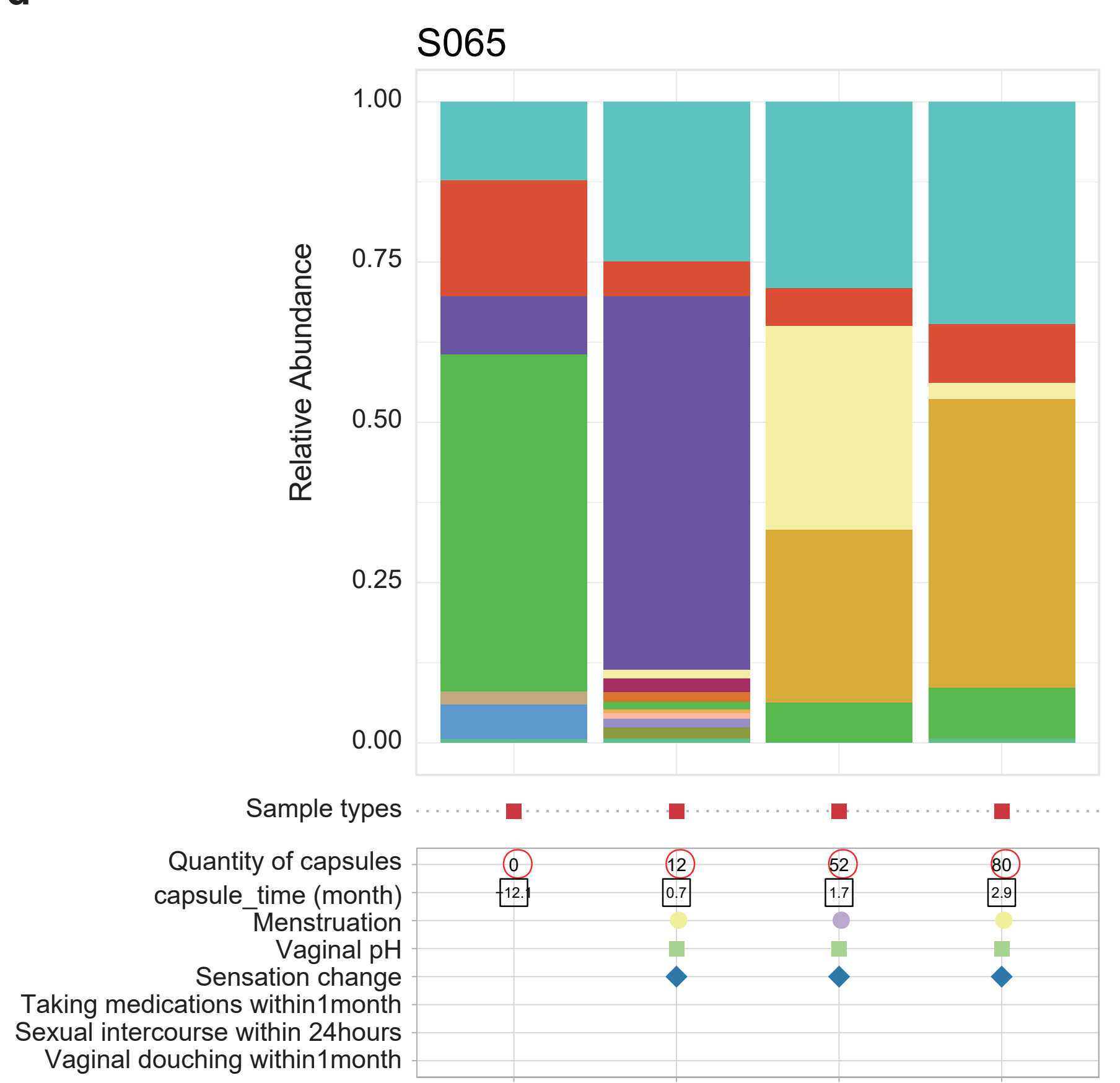

Vaginal douching within 1 month
S013
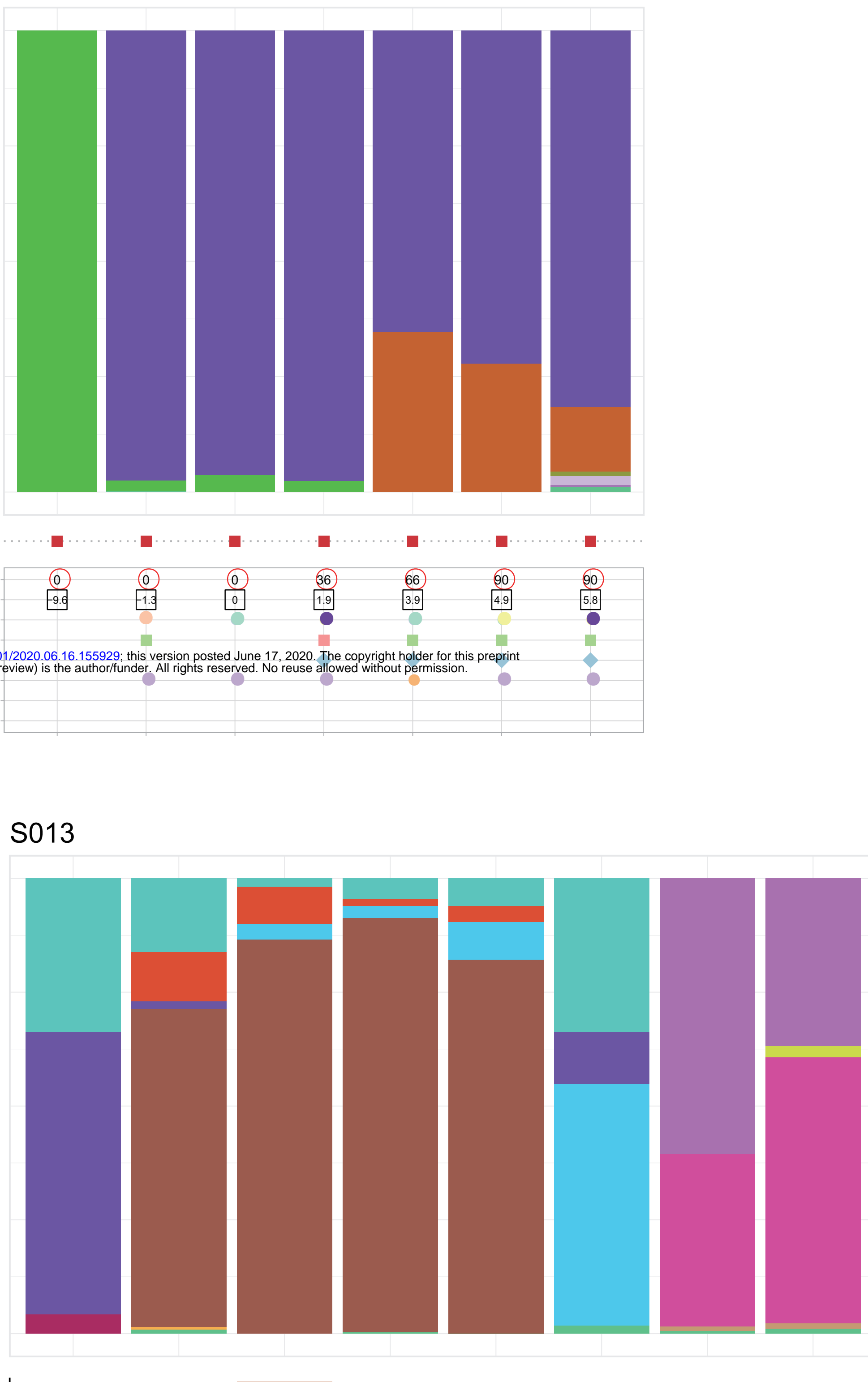